\title{
Suppression subtractive hybridization reveals differentially expressed genes in supraspinous ligaments of patients with ankylosing spondylitis
}

\author{
YING ZHANG，XU HU，CHAO ZHANG，YUE ZHOU and TONG-WEI CHU
}

Department of Orthopedics, Xinqiao Hospital, The Third Military Medical University, Chongqing 400037, P.R. China

Received April 10, 2014; Accepted December 12, 2014

DOI: $10.3892 / \mathrm{mmr} .2015 .3272$

\begin{abstract}
Ankylosing spondylitis (AS) is a severe chronic inflammatory disease that may ultimately result in the development of a 'bamboo-like' spine. Although the pathological changes that occur in AS have been extensively investigated, the mechanism underlying spinal fusion during AS remains elusive. Differentially expressed genes (DEGs) in paraspinal tissues from patients with AS compared with those from healthy controls were therefore investigated. Polymerase chain reaction (PCR)-based suppression subtractive hybridization was performed using total mRNA from the supraspinal ligaments of three patients with AS and three patients with spinal fractures as controls. From this, 27 genes were identified in all of the three independent forward libraries, which were defined as DEGs associated with AS. Reverse transcription-quantitative PCR demonstrated that six DEGs were overexpressed in the tissues from patients with AS compared with those from individuals in the control group, including those encoding transforming growth factor $\beta$ types I and III receptor, vascular endothelial growth factor, matrix metalloproteinase-3, core-binding factor $\alpha 1$ and bone morphogenetic protein 2 . Western blot analysis showed increased expression in all six of these proteins in the samples from patients with AS compared with those in the control groups. These findings suggested that changes in the expression of these genes and proteins are associated with the development of spinal fusion during the pathogenesis of AS. Furthermore, these genes may be novel markers of the risk of developing AS, in addition to being targets for the treatment of this disease.
\end{abstract}

Correspondence to: Professor Tong-Wei Chu, Department of Orthopedics, Xinqiao Hospital, The Third Military Medical University, 183 Xinqiao Road, Chongqing 400037, P.R. China E-mail: chutw@sina.com

Key words: ankylosing spondylitis, supraspinous ligament, suppression subtractive hybridization, differentially expressed genes

\section{Introduction}

Ankylosing spondylitis (AS) is a severe chronic inflammatory disease that affects the sacroiliac joints and axial skeleton. AS is characterized primarily by inflammatory back pain, which may lead to structural and functional impairment, and ultimately the development of a 'bamboo-like' spine (1-3). A significant proportion of AS patients are young adults. As there is currently no effective cure for this disease, morbidity from AS results in a high burden on families, as well as society in general $(4,5)$.

The pathogenesis of AS is complex and remains poorly understood. Hereditary factors are hypothesized to be the primary etiological agent, although infection, metabolic disorders and autoimmune disorders may also be involved $(3,6)$. The gene encoding human leukocyte antigen B27 (HLA-B27) is understood to be the most important genetic factor associated with AS (7). However, additional genes associated with an increased susceptibility to this disease have been identified. These include genes encoding HLA-B60 (8), killer cell immunoglobulin-like receptors (9), interleukin family members $(10,11)$ and tumor necrosis factor $\alpha$ (TNF- $\alpha)(12)$. However, these genetic alterations do not fully explain the pathogenesis of AS, in particular uncontrolled bone formation and subsequent spinal fusion.

Uncontrolled bone formation occurs during the pathogenesis of AS, resulting in spinal fusion and disability (4). Thus, spinal fusion is thought to be the most serious consequence of AS $(13,14)$. A previous study identified alterations in the supraspinal ligaments of patients with AS, including irregular arrangements of collagen fibrils, degeneration of fibroblasts, significant increases in microvessel density and calcification of tissue (15). This phenotype suggests a tendency to osteogenesis in the supraspinal ligaments of those with AS, indicating that further investigation of the molecular changes present in supraspinal ligaments may improve understanding of the pathogenesis of spinal fusion in patients with AS.

In order to assess the molecular changes associated with spinal fusion, polymerase chain reaction (PCR)-based suppression subtractive hybridization ( $\mathrm{SSH}$ ) was conducted, using supraspinal ligaments of patients with AS and from healthy controls. Differentially expressed genes (DEGs) between the two groups were identified, and the mRNA and protein expression patterns of six DEGs were determined. 


\section{Materials and methods}

Clinical samples. Paraspinal tissues were obtained via spinal orthopedic surgery from six patients (four males and two females; mean age, 30.2 years) who were in the active stage of AS, according to the criteria described by the American Rheumatism Association (16). The X-ray imaging of patients were carried out with a Definium 6000 (GE Healthcare, Wauwatosa, WI, USA). Paraspinal tissues were also obtained during reattachment surgery from three patients (two males and one female; mean age, 31.5 years) who had sustained spinal fractures. Supraspinous ligaments were carefully isolated from these tissue samples, washed with phosphate-buffered saline (0.01 mM, pH 7.2; Sangon Biotech Co., Ltd, Shaghai, China) and then immediately stored in liquid nitrogen, or fixed in 4\% paraformaldehyde solution (Sangon Biotech Co., Ltd) and embedded in paraffin (Sangon Biotech Co., Ltd). The study protocol was approved by the ethics committee of Xinqiao Hospital (Chongqing, China). All patients provided written informed consent for the experimental use of the obtained materials.

Pathological assessment. Pathological assessment was performed as previously described $(1,17)$. Briefly, paraffin-embedded supraspinous ligaments were cut into thin slices and stained with hematoxylin and eosin (Sangon Biotech Co., Ltd). Following dehydration, transparency and drying, all sections were evaluated by a pathologist who was blinded to patient diagnosis using a microscope (IX71, Olympus Corp., Tokyo, Japan) and Analysis FIVE software version 5.0 (Olympus Corp.).

$S S H$. Frozen tissue samples were ground in liquid nitrogen and total RNA was extracted using RNAiso plus (Takara, Dalian, China). PolyA ${ }^{+}$mRNA was isolated from total RNA using PolyATract kits (Promega Corp., Madison, WI, USA). A 2- $\mu \mathrm{g}$ aliquot of each mRNA sample was used as a template in order to generate double-stranded cDNA (dscDNA) using a SMART PCR cDNA synthesis kit (Clontech, Palo Alto, CA, USA).

SSH was performed using PCR-select cDNA subtraction kits (Clontech) according to the manufacturer's instructions. Three independent SSH experiments using the AS and control samples were conducted. Briefly, forward and reverse SSH were simultaneously performed in each independent SSH experiment. During forward SSH, the dscDNA of the AS samples was set as the Tester and the dscDNA of the control samples as the Driver (Tester and Driver were set according to the instructions). For reverse SSH, the Tester and Driver dscDNAs were exchanged. Each dscDNA was digested with RsaI (Clontech), and the blunt-ended products were ligated with adaptor. The Tester and Driver were hybridized twice, with the product used as a PCR template in order to amplify differentially expressed cDNAs. Throughout the SSH procedure, the efficiency of dscDNA synthesis, RsaI digestion, adaptor ligation and subtractive hybridization were monitored according to the manufacturer's instructions.

Construction of subtracted cDNA libraries. Differentially expressed cDNAs synthesized by SSH were recycled, inserted into a pMD-19T vector (Takara) and subsequently used to transform competent Escherichia coli DH5 $\alpha$ (Takara). The transformed cells were selected using Luria-Bertani agar plates containing ampicillin, X-gal and IPTG (Sangon Biotech Co., Ltd). Colonies containing inserts (white colonies) were assayed by colony PCR using the following procedure: Denature at $94^{\circ} \mathrm{C}$ for $5 \mathrm{~min}$, followed with 30 cycles $\left(94^{\circ} \mathrm{C}\right.$ for $30 \mathrm{sec}, 58^{\circ} \mathrm{C}$ for $30 \mathrm{sec}$, and $72^{\circ} \mathrm{C}$ for $\left.30 \mathrm{sec}\right)$. The primer pair was as follows: Nested PCR primer 1, 5'-TCGAGCGGCCGCCCGGGCAGGT-3' and nested PCR primer 2R, 5'-AGCGTGGTCGCGGCCGAGGT-3' (Clontech). The PCR products were electrophoresed on agarose gels to confirm the presence and size of the inserts prior to sequencing.

Sequencing analysis. Differentially expressed cDNA fragments from subtracted cDNA libraries were sequenced by Invitrogen Life Technologies (Shanghai, China) and the sequences were evaluated using Chromas software. Contig Express (New York, NY, USA) performed contig assembly and the obtained sequences were analyzed using the basic local alignment search tool for nucleotides (BLASTn; www.blast.ncbi.nlm.nih.gov) to search for homologous sequences. Kyoto Encyclopedia of Genes and Genomes (KEGG; http://www.genome.jp/kegg/ pathway analysis was performed in order to identify the function of the relevant genes. Only genes which were present in the results of all three independent SSH experiments were defined as DEGs and used for subsequent experiments.

Validation of mRNA expression. Total RNA samples were reverse-transcribed using the PrimeScript RT reagent kit (Takara) and cDNA samples were used as templates for reverse transrciption-quantitative PCR (RT-qPCR) using the primers listed in Table II. RT-qPCR was performed using a CFX Connect ${ }^{\mathrm{TM}}$ Cycler with SsoAdvanced ${ }^{\mathrm{TM}}$ SYBR Green supermix (Bio-Rad Laboratories, Inc., Hercules, CA, USA) according to the manufacturer's instructions. The expression levels of each DEG were normalized to those of $\beta$-actin mRNA and calculated using the $2^{-\Delta \Delta C T}$ method with SPSS version 13.0 (SPSS, Inc., Chicago, IL, USA) as previously described (18).

Western blot analysis. Total proteins were extracted from frozen tissue samples using IP Cell Lysis Reagent (Beyotime, Haimen, Jiangsu, China). Proteins were separated by $10 \%$ SDS-PAGE and transferred to polyvinylidene difluoride membranes (EMD Millipore, Billerica, MA, USA). The membranes were blocked at $37^{\circ} \mathrm{C}$ with $5 \%$ non-fat milk (Beyotime) and incubated overnight with 1:1,000 dilutions (Beyotime) of polyclonal rabbit-derived antibodies against human $\beta$-actin and vascular endothelial growth factor (VEGF; both from Beyotime), human bone morphogenetic protein 2 (BMP-2) and matrix metalloproteinase-3 (MMP-3; both from Santa Cruz Biotechnology, Inc., Dallas, TX, USA) and core binding factor- $\alpha 1$ (Cbf- $\alpha 1)$, human transforming growth factor- $\beta$ type I (TBRI) and TBRIII (all from Cell Signaling Technology, Inc., Danvers, MA, USA). The blots were washed with PBS containing 0.5\% Tween-20 (Sangon Biotech Co., Ltd) and incubated with secondary goat anti-rabbit or anti-mouse immunoglobulin $\mathrm{G}$ antibodies (Beyotime; 1:10,000). Protein bands were visualized with the SuperSignal West Pico chemiluminescence substrate 
Table I. DEGs in AS supraspinous ligaments.

\begin{tabular}{|c|c|c|c|c|c|}
\hline DEG ID & $\begin{array}{l}\text { Length } \\
\text { (bp) }\end{array}$ & $\begin{array}{c}\text { No. } \\
\text { of ESTs }\end{array}$ & Best hits & Identity & Annotation \\
\hline FL01 & 224 & 3 & NM_000576.2 & $100 \%$ & Interleukin 1 , beta \\
\hline FL02 & 218 & 14 & NM_005517.3 & $100 \%$ & High mobility group nucleosomal binding domain 2 \\
\hline FL03 & 529 & 8 & XM_005260402.1 & $100 \%$ & G protein, alpha stimulating activity polypeptide 1 \\
\hline FL04 & 213 & 22 & XM_005264576.1 & $99 \%$ & Calmodulin 2 (phosphorylase kinase delta) \\
\hline FL05 & 234 & 2 & XM_005249363.1 & $100 \%$ & Vascular endothelial growth factor A \\
\hline FL06 & 399 & 1 & NM_001568.2 & $100 \%$ & Eukaryotic translation initiation factor 3 , subunit $\mathrm{E}$ \\
\hline FL07 & 706 & 8 & NM_002298.4 & $99 \%$ & Lymphocyte cytosolic protein 1 \\
\hline FL08 & 494 & 1 & XM_005274125.1 & $100 \%$ & Carnosine synthase 1 \\
\hline FL09 & 725 & 6 & NM_001731.2 & $99 \%$ & B-cell translocation gene 1 \\
\hline FL10 & 833 & 5 & NM_000090.3 & $100 \%$ & Collagen, type III, alpha 1 \\
\hline FL11 & 552 & 15 & NM_001161727.1 & $98 \%$ & Phospholipase A2, group IIA \\
\hline FL12 & 839 & 3 & NM_003380.3 & $99 \%$ & Vimentin \\
\hline FL13 & 803 & 5 & NM_001663.3 & $100 \%$ & ADP-ribosylation factor 6 \\
\hline FL14 & 211 & 1 & NM_002966.2 & $100 \%$ & S100 calcium binding protein A10 \\
\hline FL15 & 556 & 2 & NM_000840.2 & $93 \%$ & Glutamate receptor, metabotropic 3 \\
\hline FL16 & 396 & 1 & XM_005252150.1 & $99 \%$ & Transforming growth factor, beta receptor I \\
\hline FL17 & 731 & 2 & XM_005248668.1 & $100 \%$ & Eukaryotic translation elongation factor 1 alpha 1 \\
\hline FL18 & 367 & 1 & XM_005270519.1 & $99 \%$ & Interleukin 23 receptor \\
\hline FL19 & 180 & 3 & NM_000594.3 & $100 \%$ & Tumor necrosis factor alpha \\
\hline FL20 & 578 & 1 & XM_005274246.1 & $99 \%$ & AHNAK nucleoprotein \\
\hline FL21 & 118 & 1 & NM_001200.2 & $100 \%$ & Bone morphogenetic protein 2 \\
\hline FL22 & 176 & 3 & NM_002422.3 & $99 \%$ & Matrix metalloproteinase 3 \\
\hline FL23 & 429 & 1 & NM_012232.5 & $99 \%$ & Polymerase I and transcript release factor \\
\hline FL24 & 214 & 1 & HUMCBFA & $99 \%$ & Core-binding factor, runt domain, alpha subunit 1 \\
\hline FL25 & 67 & 1 & XM_005252909.1 & $98 \%$ & Interferon regulatory factor 7 \\
\hline FL26 & 459 & 1 & NM_002933.4 & $99 \%$ & Ribonuclease, RNase A family, 1 \\
\hline FL27 & 729 & 1 & NM_001195683.1 & $99 \%$ & Transforming growth factor, beta receptor III \\
\hline
\end{tabular}

DEG, differentially expressed gene; AS, ankylosing spondylitis; ESTs, expressed sequence tags; AHNAK, desmoyokin.

(Thermo Pierce, Rockford, IL, USA) using a ChemiDoc XRS Gel Imaging System (Bio-Rad Laboratories, Inc.).

Statistical analysis. The levels of mRNA expression are presented as the mean \pm standard deviation and were compared using Student's t tests using SPSS version 13.0 (SPSS, Inc.). $\mathrm{P}<0.05$ was considered to indicate a statistically significant difference.

\section{Results}

Pathological changes in supraspinal ligaments. Pelvic X-ray images of a patient with AS demonstrated typical spinal fusion (Fig. 1A). The pathological changes in the supraspinal ligaments of patients with AS, which were subsequently used in the SSH experiments, and in control patients with spinal fractures were examined by light microscopy. Histological examination demonstrated that the collagen fibrils of control ligaments were regularly arranged and filled with diffuse extracellular matrix, with spindle-like fibroblasts running parallel to these fibrils (Fig. 1B). By contrast, the collagen fibrils of ligaments from patients with AS were irregularly organized and did not contain extracellular matrix. Furthermore, fewer fibrils were observed in these samples, in which there was also evidence of hyaline degeneration (Fig. 1C).

Efficiency of SSH. The efficiency of each SSH experiment involving glyceraldehyde 3-phosphate dehydrogenase $(G 3 P D H)$ cDNA, was tested by quantitative comparison of subtracted and unsubtracted cDNA. PCR products were detectable following 25 cycles in the AS unsubtracted sample, compared with $>10$ cycles in the forward subtracted sample (Fig. 2A). Similarly, G3PDH PCR products were detected following 25 cycles in the unsubtracted control sample, whereas $>10$ cycles were required in the reverse subtracted sample (Fig. 2B).

Characterization of the subtracted cDNA libraries. Two cDNA libraries, forward and reverse, were constructed by PCR-based SSH from the supraspinal ligaments of patients with AS and control patients in each independent SSH experiment. On 
Table II. Primers used for reverse transcription-quantitative polymerase chain reaction.

\begin{tabular}{lllc}
\hline & \multicolumn{3}{c}{ Primer sequence } \\
\cline { 2 - 3 } Gene & \multicolumn{1}{c}{ Forward $\left(5^{\prime} \rightarrow 3^{\prime}\right)$} & \multicolumn{1}{c}{ Reverse $\left(5^{\prime} \rightarrow 3^{\prime}\right)$} & Product size (bp) \\
\hline TBRI & AATGGGCTTAGTATTCTGGG & TTTCTTCAACTGATGGGTCA & 113 \\
TBRIII & TTTTGGTGTCTGAGGGTTCT & TGCTATCTTGAGTTCGGTGA & 161 \\
VEGF & TGCCCACTGAGGAGTCCAAC & ACAAATGCTTTCTCCGCTCT & 177 \\
MMP-3 & GGCAGTTTGCTCAGCCTATC & TCCAGAGTGTCGGAGTCCAG & 219 \\
Cbf- $\alpha 1$ & CTCTTCCCAAAGCCAGAGTG & ATCAGCGTCAACACCATCAT & 208 \\
BMP-2 & CCGCTGTCTTCTAGCGTTGC & CTCGTCAGAGGGCTGGGATG & 130 \\
$\beta$-actin & GAGACCTTCAACACCCCAGC & ATGTCACGCACGATTTCCC & 263 \\
\hline
\end{tabular}

TBRI/III, transforming growth factor $\beta$ type I/III; VEGF, vascular endothelial growth factor; MMP-3, matrix metalloproteinase-3; Cbf- $\alpha 1$, core binding factor- $\alpha 1$; BMP-2, bone morphogenetic protein 2 .

A

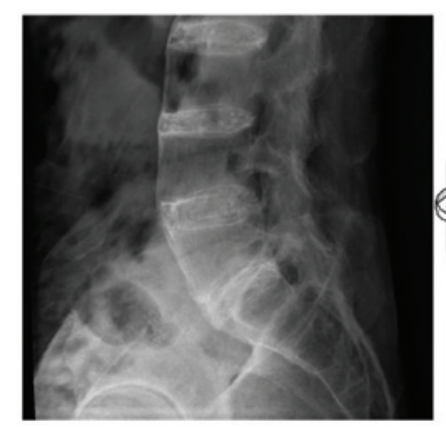

B
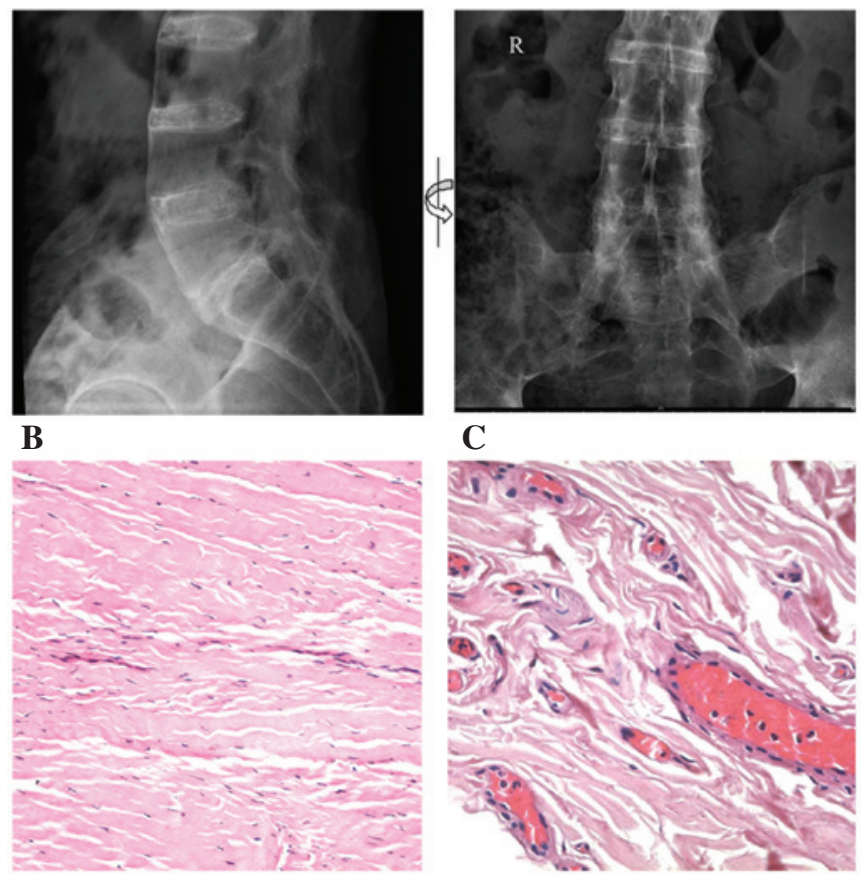

C

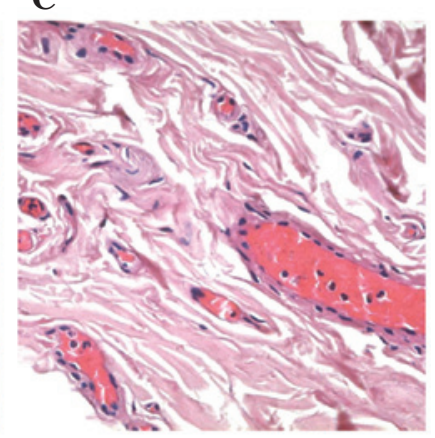

Figure 1. Pathological features of supraspinous ligaments obtained from patients with AS. (A) X-ray of a spine and sacroiliac joint with joint fusion from a patient with AS. 'R' indicates the right side. (B) Supraspinal ligaments obtained from patients who had sustained spinal fractures (control). (C) Supraspinal ligaments from patients with AS. Samples in B and C were stained with hematoxylin and eosin and examined by light microscopy (magnification, x200). AS, ankylosing spondylitis.

average, 200 differentially expressed cDNA fragments from the forward subtracted cDNA library and $\sim 150$ from the reverse library were identified in each SSH experiment. Prior to sequencing, all clones were isolated and assayed by colony PCR for the presence of cDNA inserts and in order to estimate the size of each product. In the SSH experiments under optimized conditions, a total of 212 clones, 153 from the forward and 59 from the reverse libraries, were isolated and sequenced, with insert sizes ranging from 67 to $839 \mathrm{bp}$. Following removal of the vector sequences and low-quality expressed sequence tags (ESTs), the average available sequence was $\sim 319$ bp in length.
A

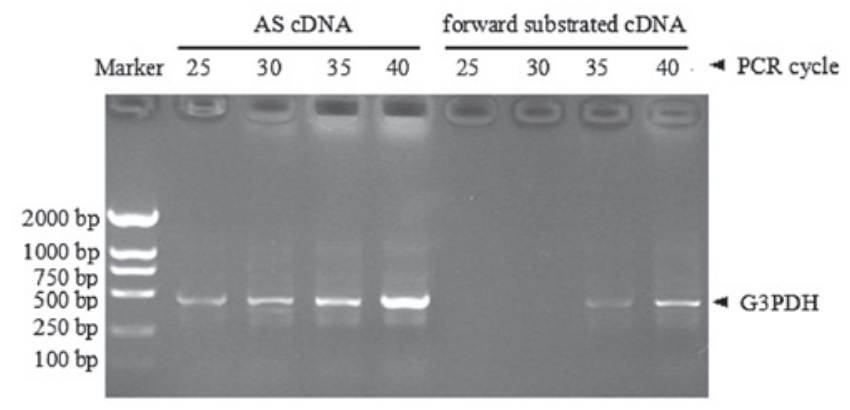

B

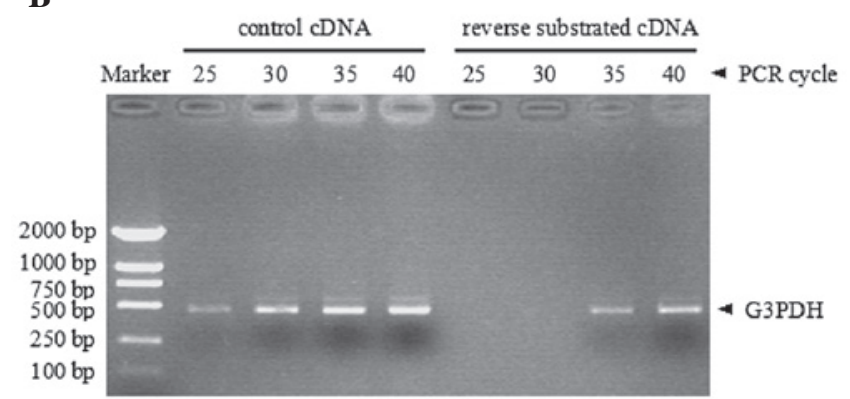

Figure 2. Evaluation of subtraction efficiency. (A) Reduction of G3PDH abundance in the forward SSH sample. Subtracted AS-control cDNA and unsubtracted AS cDNA were used as PCR templates. Data from one of three independent experiments is shown. (B) Reduction of G3PDH abundance in the reverse SSH sample. Subtracted control-AS cDNA and unsubtracted control cDNA were used as PCR templates. Data from one of three independent experiments is shown. G3PDH; glyceraldehyde 3-phosphate dehydrogenase; $\mathrm{SSH}$, suppressive subtractive hybridization; PCR, polymerase chain reaction; AS, ankylosing spondylitis.

The cleaned ESTs of each SSH experiment were subsequently assembled. Using the BLASTn tool, all sequences from forward or reverse libraries demonstrated significant similarity with genes from the GenBank database (http://www.ncbi.nlm. nih.gov/genbank/). A total of 27 DEGs were presented in all of the three forward libraries. All of these were functionally annotated in the KEGG; http://www.genome.jp/kegg/ online server. These genes are thus candidate DEGs associated with AS, and are listed in Table I. 

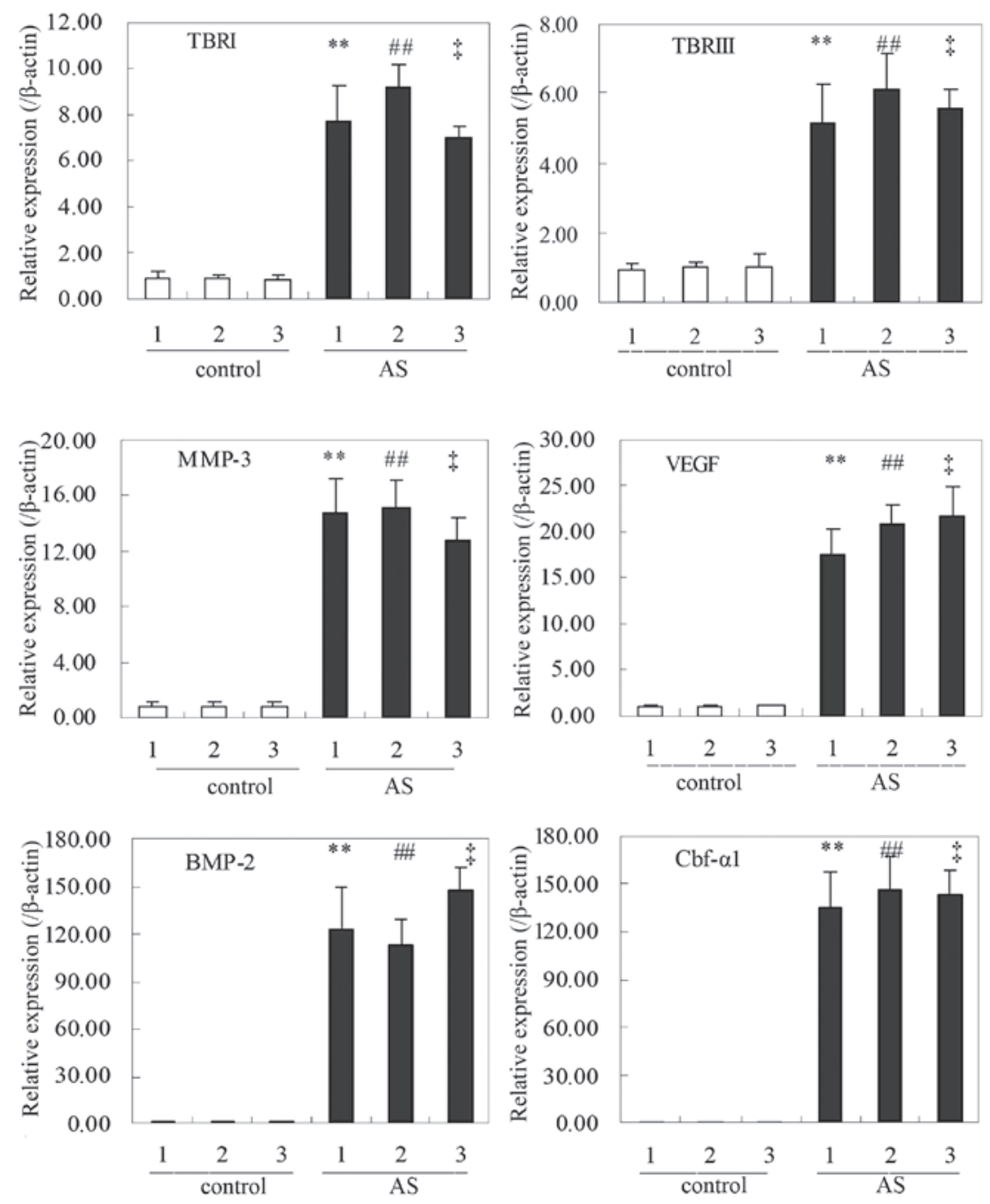

Figure 3. Expression of selected differentially expressed genes in supraspinal ligaments from patients with ankylosing spondylitis. The templates were the same as those used for the suppressive subtractive hybridization experiments. Expression levels of each gene were normalized to those of $\beta$-actin in the same sample and calculated using the $2^{-\Delta \Delta C \mathrm{CT}}$ method. Values are presented as the mean \pm standard deviation of three experimental repeats. ${ }^{* *} \mathrm{P}<0.01 \mathrm{vs}$. control-1, ${ }^{\# \#} \mathrm{P}<0.01$ vs. control-2, ${ }^{\mathrm{t}} \mathrm{P}<0.01$ vs. control-3. TBRI/III, transforming growth factor $\beta$ type I/III; VEGF, vascular endothelial growth factor; MMP-3, matrix metalloproteinase- 3 ; Cbf- $\alpha 1$, core binding factor- $\alpha 1$; BMP-2, bone morphogenetic protein 2.

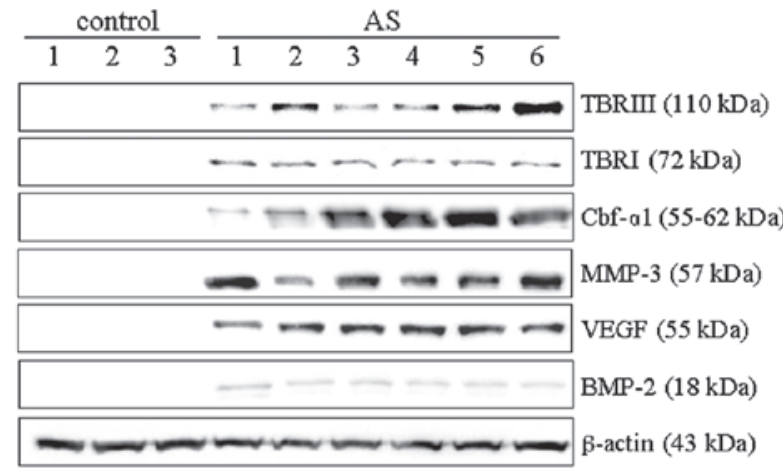

Figure 4. Quantitative western blot analyses of identified differentially expressed genes in supraspinal ligaments from patients with AS and control subjects. Total protein was extracted from supraspinal ligaments of six patients with AS and three patients with spinal fractures. Equal quantities of total protein were loaded onto each lane and the subsequent blots probed with antibodies to TBRI, TBRIII, Cbf- $\alpha 1$, MMP-3, VEGF and BMP-2, and normalized relative to the expression of $\beta$-actin. A blot representative of three independent experiments is shown. TBRI/III, transforming growth factor $\beta$ type I/III; VEGF, vascular endothelial growth factor; MMP-3, matrix metalloproteinase-3; Cbf- $\alpha 1$, core binding factor- $\alpha 1$; BMP-2, bone morphogenetic protein 2; AS, ankylosing spondylitis.
Confirmation of differentially expressed $m R N A$. In order to confirm the differential gene expression pattern in supraspinous ligaments from patients with AS, six upregulated DEGs were selected based on functional annotations. Although inflammation-associated genes, including tumor necrosis factor $\alpha(\mathrm{TNF}-\alpha)$, interleukin-1 $\beta$ (IL-1 $\beta$ ) and interleukin 23 receptor (IL-23R) are hypothesized to be involved in the pathogenesis of AS $(19,20)$, the present study focused on spinal fusion rather than inflammation. Primers for these genes were designed using Primer Premier 5 software (Table II). Reverse transcription-quantitative PCR (RT-qPCR) analyses of the same AS and control samples as those used for SSH experiments were performed in order to quantify the expression of mRNAs encoded by the candidate genes. Theoretically, the expression levels of expression of each candidate gene would be higher in the samples from patients with AS than those in the samples from control patients.

RT-qPCR analyses showed that, in accordance with the results of the SSH experiments, the levels of mRNAs encoding the six proteins TBRI/III, VEGF, MMP-3, Cbf- $\alpha 1$ and BMP-2 
were significantly increased in AS samples as compared with those in the control samples (Fig. 3).

Confirmation that the encoded proteins are differentially expressed. In order to further investigate the expression of these six DEGs, western blot analysis was conducted using total protein extracted from the supraspinal ligaments of the six patients with AS and three control subjects. An increased expression of all six proteins was detected in the samples from patients with AS as compared with those in the three controls (Fig. 4). Furthermore, these proteins were not detected at all in the control samples, although their mRNA was present at low levels. These results indicated that TBRI, TBRIII, VEGF, Cbf- $\alpha 1$, BMP-2 and MMP-3 proteins are abundantly expressed in the supraspinal ligaments of patients with AS.

\section{Discussion}

Chronic inflammation, back pain, bone erosion and syndesmophyte formation are characteristic features of $\operatorname{AS}(6,21)$, with bone formation and subsequent spinal fusion being fundamental causes of AS-associated disability. Whilst histopathological changes have been observed in the spinal tissues of patients with AS $(1,5,17)$, the molecular mechanisms underlying spinal fusion remain elusive. There are currently no available methods of reversing the process of spinal fusion, with the exception of orthopedic spinal surgery.

PCR-based SSH, which is distinct from microarray technology, is a rapid and sensitive method for the identification of DEGs. SSH directly provides partial sequences of DEGs, including scarce or novel genes. Genes that were differentially expressed in the supraspinous ligaments of AS compared with those of control patients were identified using SSH. The three forward libraries yielded 27 genes defined as DEGs in the AS samples. While the association between HLA-B27 and AS was identified in patients aged $>30$ years $(7)$, it was not detected in AS supraspinous ligaments via SSH. However, differential expression of inflammation-associated genes, including IL-1 $\beta$, TNF- $\alpha$ and IL-23R, which are hypothesized to be associated with the development of AS $(20,22)$, was detected. RT-qPCR and western blot analyses identified six candidate proteins, TBRI, TBRIII, VEGF, MMP-3, Cbf- $\alpha 1$ and BMP-2, as being associated with AS, but not inflammation, and contributing to spinal fusion.

Members of the TGF- $\beta$ superfamily are known to be important regulators of cell proliferation, differentiation, morphogenesis, apoptosis cancer and heritable disorders (23). TGF- $\beta$ was shown to promote downstream signaling pathways through three cell surface receptors: TBRI, TBRII and TBRIII. TBRI and TBRII were identified as serine/threonine kinases. Following ligand binding, TBRI recruits TBRII and the two receptors form a stable heteromeric complex on the cell surface. TBRII phosphorylates and activates TBRI (24). TBRIII is an abundant cell surface proteoglycan, characterized as a ubiquitously-expressed transmembrane receptor that acts as a TGF- $\beta$ and inhibin receptor (25). TBRIII has a large extracellular domain, which tightly binds TGF- $\beta$ with a high affinity (26). TBRIII regulates TGF- $\beta$ signaling by presenting it to the activated TBRI/TBRII complex. The present study demonstrated that TBRI and TBRIII were highly expressed in the supraspinal ligaments of patients with AS, compared with those from control patients, suggesting that there is excessive activation of TGF- $\beta$ signaling within AS tissues. This increase in activation may contribute to uncontrolled bone formation and spinal fusion.

TBRIII also acts as a BMP-2 co-receptor, increasing the quantity of BMP-2 that binds to the BMP signaling receptors, activin-like receptor kinases 3 (ALK3) and ALK6 (26). BMP-2 is an essential regulator of bone development and facilitates osteoblastic differentiation and bone formation (26). Of note, BMP-2 and TBRIII were highly expressed in all of the AS samples examined, indicating that excess activation of the BMP-2/TBRIII pathway may be a bone morphogenetic mechanism that contributes to spinal fusion in patients with AS.

Cbf- $\alpha 1$ is an important osteoblast-specific transcription factor that may differentially activate osteoblasts during embryonic development (27). In the absence of Cbf- $\alpha 1$, osteoblast differentiation and bone development have been shown to be limited (28). The present study demonstrated that the levels of Cbf- $\alpha 1$ mRNA and protein were markedly higher in AS than those in control samples, suggesting that Cbf- $\alpha 1$ may contribute to spinal fusion in patients with AS.

During skeletal development, bone formation is known to be coupled to angiogenesis (29). VEGF is an endothelial cell growth factor, which is an early marker of angiogenesis and also a marker of bone formation (30). The present study showed that VEGF expression was higher in the samples from patients with AS than that in control samples, indicating that VEGF may also contribute to spinal fusion.

The present study demonstrated that the expression of MMP-3 mRNA and protein was higher in AS samples than that in control samples. MMP-3 has been shown to be an important mediator of bone matrix and cartilage damage (31). Recent reports have suggested that MMP-3 may also serve as a biomarker with which to assess disease activity in patients with AS (32), and for monitoring the response of patients to drug treatment in clinical practice (33). Damage to articular surfaces and cartilage that occurs in spinal joints are considered to be the significant degenerative changes during the early pathogenesis of spinal fusion (3). Although, to the best of our knowledge, there is no evidence to suggest that MMP-3 primes bone formation, it may participate in the process of spinal joint damage, thereby contributing to spinal fusion during the development of AS.

In conclusion, the present study isolated six DEGs, namely TBRI, TBRIII, VEGF, MMP-3, Cbf- $\alpha 1$ and BMP-2, in the supraspinal ligaments of patients with AS. These six DEGs were hypothesized to be potential osteogenic factors associated with excessive bone formation during AS. Further investigations are required in order to determine whether any of these genes participate in bone formation and subsequent spinal fusion during the pathogenesis of AS.

\section{Acknowledgements}

This study was supported by the National Natural Science Foundation of China (grant no. 81071523) and the Natural Science Foundation of Chongqing (grant no. CSTC 
2011BA5009). The authors would like to thank the staff and patients at the Department of Orthopaedics of Xinqiao Hospital (Chongqing, China) who participated in this study.

\section{References}

1. Appel H, Kuhne M, Spiekermann S, et al: Immunohistochemical analysis of hip arthritis in ankylosing spondylitis: evaluation of the bone-cartilage interface and subchondral bone marrow. Arthritis Rheum 54: 1805-1813, 2006.

2. Chou CH, Lin MC, Peng CL, et al: A nationwide population-based retrospective cohort study: increased risk of acute coronary syndrome in patients with ankylosing spondylitis Scand J Rheumatol 43: 132-136, 2014.

3. Braun J and Sieper J: Ankylosing spondylitis. Lancet 369 1379-1390, 2007.

4. Thomas GP and Brown MA: Genomics of ankylosing spondylitis. Discov Med 10: 263-271, 2010.

5. Lories RJ and Baeten DL: Differences in pathophysiology between rheumatoid arthritis and ankylosing spondylitis. Clin Exp Rheumatol 27 (Suppl 55): S10-S14, 2009.

6. Tam LS, Gu J and Yu D: Pathogenesis of ankylosing spondylitis. Nat Rev Rheumatol 6: 399-405, 2010.

7. Khan MA, Mathieu A, Sorrentino R and Akkoc N: The pathogenetic role of HLA-B27 and its subtypes. Autoimmun Rev 6 : 183-189, 2007.

8. Wei JC, Tsai WC, Lin HS, Tsai CY and Chou CT: HLA-B60 and B61 are strongly associated with ankylosing spondylitis in HLA-B27-negative Taiwan Chinese patients. Rheumatology (Oxford) 43: 839-842, 2004.

9. Diaz-Peña R, Blanco-Gelaz MA, Suárez-Alvarez B, et al: Activating KIR genes are associated with ankylosing spondylitis in Asian populations. Hum Immunol 69: 437-442, 2008.

10. Kim TH, Stone MA, Rahman P, et al: Interleukin 1 and nuclear factor-kappaB polymorphisms in ankylosing spondylitis in Canada and Korea. J Rheumatol 32: 1907-1910, 2005.

11. McGarry F, Neilly J, Anderson N, Sturrock R and Field M: A polymorphism within the interleukin 1 receptor antagonist (IL-1Ra) gene is associated with ankylosing spondylitis. Rheumatology (Oxford) 40: 1359-1364, 2001.

12. van Sijl AM, van Eijk IC, Peters MJ, et al: Tumour necrosis factor blocking agents and progression of subclinical atherosclerosis in patients with ankylosing spondylitis. Ann Rheum Dis, 2013, (Epub ahead of print).

13. Feldtkeller $E$ and Braun J: Impact of sex on inheritance of ankylosing spondylitis. Lancet 355: 1096-1097; author reply 1098, 2000

14. Davey-Ranasinghe $\mathrm{N}$ and Deodhar A: Osteoporosis and vertebral fractures in ankylosing spondylitis. Curr Opin Rheumatol 25 509-516, 2013

15. Xu HF, Zhang L, Zhang Y Chu TW: Histopathologicai and ultrastructural observation of the supraspinal ligaments in ankylosing spondylitis. Chinese Journal of Rheumatology 17: 152-154, 2013 (In Chinese).

16. Arnett FC, Edworthy SM, Bloch DA, et al: The American Rheumatism Association 1987 revised criteria for the classification of rheumatoid arthritis. Arthritis Rheum 31: 315-324, 1988.
17. Appel H, Loddenkemper C, Grozdanovic Z, et al: Correlation of histopathological findings and magnetic resonance imaging in the spine of patients with ankylosing spondylitis. Arthritis Res Ther 8: R143, 2006.

18. Pan X, Yue J, Ding G, et al: Leucine-rich repeat 11 of Toll-like receptor 9 can tightly bind to $\mathrm{CpG}$-containing oligodeoxynucleotides, and the positively charged residues are critical for the high affinity. J Biol Chem 287: 30596-30609, 2012.

19. Stone MA, Inman RD, Wright JG and Maetzel A: Validation exercise of the Ankylosing Spondylitis Assessment Study (ASAS) group response criteria in ankylosing spondylitis patients treated with biologics. Arthritis Rheum 51: 316-320, 2004.

20. Hreggvidsdottir HS, Noordenbos T and Baeten DL: Inflammatory pathways in spondyloarthritis. Molecular immunology 57: 28-37, 2014.

21. Gratacós J, Collado A, Filella X, et al: Serum cytokines (IL-6, TNF-alpha, IL-1 beta and IFN-gamma) in ankylosing spondylitis: a close correlation between serum IL-6 and disease activity and severity. Br J Rheumatol 33: 927-931, 1994.

22. Stone MA and Inman RD: The genetics of cytokines in ankylosing spondylitis. J Rheumatol 28: 1203-1206, 2001.

23. Massagué J, Blain SW and Lo RS: TGFbeta signaling in growth control, cancer, and heritable disorders. Cell 103: 295-309, 2000.

24. Derynck R, Akhurst RJ and Balmain A: TGF-beta signaling in tumor suppression and cancer progression. Nat Genet 29: 117-129, 2001.

25. Blobe GC, Schiemann WP, Pepin MC, et al: Functional roles for the cytoplasmic domain of the type III transforming growth factor beta receptor in regulating transforming growth factor beta signaling. J Biol Chem 276: 24627-24637, 2001.

26. Kirkbride KC, Townsend TA, Bruinsma MW, Barnett JV and Blobe GC: Bone morphogenetic proteins signal through the transforming growth factor-beta type III receptor. J Biol Chem 283: 7628-7637, 2008.

27. Ducy P, Starbuck M, Priemel M, et al: A Cbfa1-dependent genetic pathway controls bone formation beyond embryonic development. Genes Dev 13: 1025-1036, 1999.

28. Otto F, Thornell AP, Crompton T, et al: Cbfa1, a candidate gene for cleidocranial dysplasia syndrome, is essential for osteoblast differentiation and bone development. Cell 89: 765-771, 1997.

29. Portal-Núñez S, Lozano D and Esbrit P: Role of angiogenesis on bone formation. Histol Histopathol 27: 559-566, 2012.

30. Clarkin CE and Gerstenfeld LC: VEGF and bone cell signalling: an essential vessel for communication? Cell Biochem Funct 31: $1-11,2013$.

31. Maksymowych WP: Biomarkers in spondyloarthritis. Curr Rheumatol Rep 12: 318-324, 2010.

32. Soliman E, Labib W, el-Tantawi G, Hamimy A, Alhadidy A and Aldawoudy A: Role of matrix metalloproteinase-3 (MMP-3) and magnetic resonance imaging of sacroiliitis in assessing disease activity in ankylosing spondylitis. Rheumatol Int 32: 1711-1720, 2012.

33. Arends S, van der Veer E, Groen H, et al: Serum MMP-3 level as a biomarker for monitoring and predicting response to etanercept treatment in ankylosing spondylitis. J Rheumatol 38: 1644-1650, 2011. 